\title{
CCN1 induces a reversible epithelial-mesenchymal transition in gastric epithelial cells
}

\author{
Jianyuan Chai ${ }^{1,2}$, Manith Norng ${ }^{1}$, Cristina Modak ${ }^{1}$, Kevin M Reavis ${ }^{1,3}$, Wasim Mouazzen ${ }^{1}$ and Jennifer Pham ${ }^{1}$
}

CCN1 is a matricellular protein that activates many genes related to wound healing and tissue remodeling in fibroblasts, but its effect on epithelial cells remains unclear. This study examined the role of CCN1 in epithelial wound healing using rat gastric epithelial cells and rat stomach ulcer as in vitro and in vivo models, respectively. We found that CCN1 expression is highly upregulated in the epithelial cells adjacent to a wound and remains high until the wound is healed. Upregulation of CCN1 activates a transient epithelial-mesenchymal transition in the epithelial cells at the migrating front and drives wound closure. Once the wound is healed, these epithelial cells and their progeny can resume their original epithelial phenotype. We also found that CCN1-induced E-cadherin loss is not due to transcriptional regulation but rather protein degradation due to the collapse of adherens junctions, which is contributed by $\beta$-catenin translocation.

CCN1-activated integrin-linked kinase mediates this process. Finally, our in vivo study showed that locally neutralizing CCN1 drastically impairs wound closure, whereas local injection of recombinant CCN1 protein induces expression of vimentin and smooth muscle $\alpha$-actin in normal gastric mucosal epithelial cells and accelerates re-epithelialization during ulcer healing. In conclusion, our study indicates that CCN1 can induce reversible epithelial-mesenchymal transition, and this feature may have great value for clinical wound healing.

Laboratory Investigation (2010) 90, 1140-1151; doi:10.1038/labinvest.2010.101; published online 10 May 2010

KEYWORDS: CCN1; EMT; wound healing; E-cadherin; $\beta$-catenin; ILK

Normal epithelium is maintained by cell-cell interactions including tight junctions, adherens junctions, gap junctions, and desmosomes, among which adherens junctions have a pivotal role in regulating the activity of the entire junctional complex. Adherens junctions contain E-cadherin in complexes with $\alpha$ - and $\beta$-catenin. The stability of this core complex is critical to the integrity of the epithelial barrier. However, under certain physiological or pathological conditions, for example embryonic development, wound healing, or cancer progression, some epithelial cells may lose their intercellular connections and obtain mesenchymal phenotype, so-called epithelial-mesenchymal transition (EMT). During EMT, adherens junctions are collapsed to free epithelial cells from the surrounding tissue. Meantime, the cytoskeleton is reorganized by expression of mesenchymal proteins, for example vimentin, fibronectin, and/or metalloproteinases (MMP), to confer them the ability to move through the extracellular matrix (ECM) to their new destinations. ${ }^{1,2}$
CCN1 is a matricellular protein that belongs to the $\mathrm{CCN}$ family, ${ }^{3,4}$ which currently consists of six members: CCN1 (Cysteine-rich 61 or Cyr61), CCN2 (connective tissue growth factor or CTGF), CCN3 (nephroblastoma-overexpressed protein or NOV), CCN4 (Wnt-inducible secretory protein-1 or WISP-1), CCN5 (Wnt-inducible secretory protein-2 or WISP-2), and CCN6 (Wnt-inducible secretory protein-3 or WISP-3). The name CCN was coined in 1993 based on the

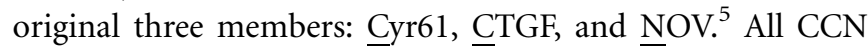
members are $30-40 \mathrm{kDa}$ secreted proteins sharing 30-50\% overall amino-acid sequence identities with one another. They are characterized by an extraordinarily high content of cysteine residues ( $>10 \%$ by mass), whose position is highly conserved within the peptide. Like immediate-early genes (eg c-fos and c-jun), CCN genes are usually expressed at a very low level in quiescent cells, but can be rapidly activated by growth factor stimulation. However, unlike the classic immediate-early genes, which are expressed only transiently (1-2 h) on stimulation, CCN genes can sustain a high level of

\footnotetext{
'Laboratory of GI Injury and Cancer, VA Long Beach Healthcare System, Long Beach, CA, USA; ${ }^{2}$ Department of Medicine, University of California, Irvine, CA, USA and ${ }^{3}$ Department of Surgery, University of California, Irvine, CA, USA Correspondence: Dr J Chai, PhD, Department of Research (09-151), VA Long Beach Healthcare System, 5901 E 7th Street, Long Beach, CA 90822, USA. E-mail: jianyuan.chai@va.gov 
expression for over a day once stimulated. Accruing evidence directs to $\mathrm{CCN}$ proteins as major players in global control over cellular activities in various tissues. ${ }^{3,4}$

The CCN1 gene (initially named as 3CH61) was discovered in 1985 as a growth factor-inducible immediateearly gene. ${ }^{6}$ Its $5^{\prime}$ flanking region contains a CArG element, a binding site for serum response factor (SRF), which has been identified in $>300$ genes ( $\sim 1 \%$ of our entire genome).$^{7}$ Our earlier studies have shown critical roles of SRF in re-epithelialization during gastric ulcer healing, ${ }^{8}$ in myofibroblast differentiation during esophageal injury repair, ${ }^{9}$ and in angiogenesis. ${ }^{10}$ The existence of a CArG element in the regulatory region of $\mathrm{CCN} 1$ is responsible for its growth factor inducibility. ${ }^{7,11}$ As a matricellular protein, $\mathrm{CCN} 1$ has features intermediate between conventional growth factors and structural ECM molecules. Unlike growth factors, CCN1 usually requires higher concentration ( $50 \mathrm{ng} / \mathrm{ml}-5 \mu \mathrm{g} / \mathrm{ml}$ ) and prolonged treatment $(>24 \mathrm{~h})$ to show an effect in cell culture, but its influence can persist for days. ${ }^{12}$ Moreover, $\mathrm{CCN} 1$ can serve regulatory roles without functioning as an integral component of structural ECM, which is the reason why $\mathrm{CCN} 1$ is often used either as a component of the cell culture bed or as a supplement to the culture medium. CCN1 also supports such cellular events as cell adhesion, proliferation, migration, differentiation, and survival-the fundamental biological processes required for wound healing. The importance of CCN1 in wound healing was further strengthened by the data generated from a microarray study in which CCN1-activated genes fall in several groups important for wound healing: ${ }^{13}$ angiogenesis (eg VEGF), inflammation (eg interleukin-1 $\beta$ ), cell-matrix interactions (eg integrin) and ECM remodeling (eg MMP).

To date, all of the known cellular activities of CCN1 are mediated through a pool of integrin receptors. ${ }^{14}$ Integrinlinked kinase (ILK), a serine/threonine kinase, has an essential role in integrin in-and-out signaling. ${ }^{15}$ Overexpression of ILK in tubular epithelial cells was found to induce dissociation of $\beta$-catenin from E-cadherin. ${ }^{16}$ ILK phosphorylates glycogen synthase kinase (GSK-3 $\beta$ ), a multifunctional serine/threonine kinase. In normal epithelial cells, GSK-3 $\beta$ keeps $\beta$-catenin phosphorylated at serine-37, thereby stabilizing adherens junctions. ${ }^{17}$ ILK-mediated serine- 9 phosphorylation and consequent inactivation of GSK-3 $\beta$ leave $\beta$-catenin dephosphorylated. As a result, $\beta$-catenin dissociates from $\alpha$-catenin with concomitant loss of E-cadherin and translocates into the nucleus, where it becomes a transcriptional activator in association with lymphoid enhancer factor (LEF-1). ${ }^{18}$ GSK-3 $\beta$ also regulates the function of Snail-a transcriptional repressor of E-cadherin. A recent study showed that Snail contains two GSK-3 $\beta$ phosphorylation motifs: phosphorylation of the first motif causes Snail to undergo degradation, whereas phosphorylation of the second motif prevents Snail from going into the nucleus, both of which relieve Snail-mediated E-cadherin transcriptional repression. ${ }^{19}$ Therefore, when GSK-3 $\beta$ is inhibited by ILK phosphorylation, this could lead to Snail activation thereby suppressing E-cadherin transcription, resulting in EMT.

The exact role of CCN1 in EMT remains unclear. One study showed that overexpression of CCN1 in glioma cells activates ILK, increases GSK- $3 \beta$ phosphorylation, and stimulates $\beta$-catenin translocation, ${ }^{20}$ suggesting a possible involvement for CCN1 in EMT. Our recent study showed a similar effect in esophageal epithelial cells. ${ }^{21}$ However, given the cell type specificity of CCN1 signaling, ${ }^{22}$ which can lead to opposing outcomes in different tissues, whether the same is true in gastric cells is still unknown.

\section{MATERIALS AND METHODS Cell Culture, Transfection, and Treatment}

Rat gastric mucosal epithelial cells (RGM1) (Riken Cell Bank, Tsukuba, Japan) were cultured in DMEM/F-12 medium (Invitrogen, Carlsbad, CA, USA) supplemented with $10 \%$ fetal bovine serum (Sigma-Aldrich, Saint Louis, MI, USA). Rat fibroblast-Rat2 cells (American Type Culture Collection, Manassas, VA, USA) were cultured in DMEM medium containing $10 \%$ fetal bovine serum and were used as a positive control for mesenchymal markers.

The following plasmids were used for transfection: pVP22 (Invitrogen) with a human CCN1 cDNA, pRS with shRNA against either CCN1 or ILK (Origene, Rockville, MD, USA), pRS with negative control shRNA, or the vectors without an insert as control. All cell transfections were done in Lipofectamine-2000 (Invitrogen) according to the manufacturer's protocol. Stable transfectants were selected by Neomycin or Puromycin (Invitrogen).

For cell treatment, RGM1 cells were plated either on sixwell-plates/Petri dishes (for RNA or protein isolation) or on collagen-coated coverslips (for staining). After $24 \mathrm{~h}$ of serum deprivation, cells were treated for the indicated time with either recombinant human CCN1 protein (Abnova, Taiwan) at $100 \mathrm{ng} / \mathrm{ml}$ or the vehicle. Medium was refreshed every other day.

\section{Real-Time RT-PCR}

Cells were cultured in six-well plates till desired confluence. Total RNA was extracted from the cells using RNeasy kit (Qiagen, Valencia, CA, USA) following the manufacturer's protocol. Reverse transcription was done in MyCycler (Bio-Rad, Hercules, CA, USA) following the protocol: $25^{\circ} \mathrm{C} /$ $10 \mathrm{~min}-55^{\circ} \mathrm{C} / 30 \mathrm{~min}-85^{\circ} \mathrm{C} / 5 \mathrm{~min}-4^{\circ} \mathrm{C} / \infty$. All of the reagents for reverse transcription, including Transcriptor Reverse Transcriptase, PCR Nucleotide Mix, Protector RNase Inhibitor, and Random Primer $\mathrm{p}[\mathrm{dN}]_{6}$, were purchased from Roche (Mannheim, Germany). Real-time PCR was performed in iCycler (Bio-Rad) following the two-step program, using SYBR Green master mix and specific primers from SABiosciences (Frederick, MD, USA). GAPDH was used as an internal control. Data were generated from at least five independent experiments and were analyzed according to 
the $\Delta \Delta \mathrm{C}_{\mathrm{t}}$ method. Briefly, $\Delta \mathrm{C}_{\mathrm{t}}$ was calculated by subtracting the $\mathrm{C}_{\mathrm{t}}$ value of GAPDH from the $\mathrm{C}_{\mathrm{t}}$ value for each gene of interest; and then $\Delta \Delta \mathrm{C}_{\mathrm{t}}$ was calculated by subtracting the $\Delta \mathrm{C}_{\mathrm{t}}$ of the control from the $\Delta \mathrm{C}_{\mathrm{t}}$ of the treatment for each gene of interest; and finally the fold change was calculated using the formula: fold change $=2^{\left(-\Delta \Delta C_{t}\right)}$. For Rat2 cells, $\Delta \Delta C_{t}$ was calculated by subtracting the $\Delta \mathrm{C}_{\mathrm{t}}$ of RGM1 cells from the $\Delta \mathrm{C}_{\mathrm{t}}$ of Rat2 cells for each gene of interest, as no treatment was given to Rat2 cells. Graphs were generated based on fold changes compared with RGM1 baseline.

\section{Western Blot Analysis}

Western blot analyses were done as described in our earlier studies. ${ }^{8-10}$ Antibodies used for western blot analysis include E-cadherin, GSK-3 $\beta$, phosphorylated serine, vimentin (BD Biosciences, San Jose, CA, USA); $\beta$-catenin, LEF-1, CCN1 (Santa Cruz Biotechnology, Santa Cruz, CA, USA); active $\beta$-catenin, ILK (Millipore, Billerica, MA, USA); Serine-37 phosphorylated $\beta$-catenin, GAPDH (Abcam, Cambridge, MA, USA); and Serine-9 phosphorylated GSK-3 $\beta$, Ubiquitin, Glutathione S-transferase (Cell Signaling, Danvers, MA, USA). Signals were quantified with the TotalLab TL100 software (Nonlinear Dynamics, Garth Heads, UK) based on at least five replicates.

\section{Immunoprecipitation and Kinase Assay}

These assays were done following our earlier protocols. ${ }^{9,23}$ Briefly, $200 \mu \mathrm{g}$ of protein lysate was adjusted to $1 \mu \mathrm{g} / \mathrm{ml}$ concentration and incubated for $2 \mathrm{~h}$ at $4^{\circ} \mathrm{C}$ with agitation with protein A-agarose beads (Millipore) coated with $2 \mu \mathrm{g}$ of specific antibody against one of the following proteins: E-cadherin, $\beta$-catenin, ILK, and GSK-3 $\beta$. The beads were washed twice in the lysis buffer. For kinase assay, the beads were washed two more times with the kinase buffer $(25 \mathrm{mM}$ Tris/pH 7.5, $5 \mathrm{mM} \beta$-glycerophosphate, $2 \mathrm{mM}$ DTT, $0.1 \mathrm{mM}$ $\mathrm{Na} 3 \mathrm{VO} 4$, and $10 \mathrm{mM} \mathrm{MgCl} 2$ ), and then incubated in $50 \mu \mathrm{l}$ of kinase buffer containing $0.2 \mathrm{mM}$ ATP and $1 \mu \mathrm{g}$ of substrate protein at $30^{\circ} \mathrm{C}$ for $30 \mathrm{~min}$. Recombinant GSK-3 $\beta$ (Cell Signaling) and $\beta$-catenin (Millipore) were used as substrates for the ILK and GSK-3 $\beta$ kinase assays, respectively.

Proteins were eventually dissociated from the beads by boiling in SDS protein sample buffer for $5 \mathrm{~min}$, separated by western blotting and probed with indicated antibodies.

\section{Immunofluorescence/Immunocytochemistry}

As described in our earlier studies, ${ }^{8,23}$ cells were cultured till desired confluence on microscopic coverslips that were precoated with $5 \%$ rat tail type I collagen (BD Biosciences) in $0.02 \mathrm{~N}$ acetic acid. After serum starvation and indicated treatment, cells were fixed for $10 \mathrm{~min}$ in either $4 \%$ paraformaldehyde (for membrane-associated antigens) or cold methanol (for cytoplasmic antigens). For cytoplasmic antigens, cells were also permeabilized in cold acetone for $5 \mathrm{~min}$ after fixation. Before a primary antibody was applied, cells were incubated in serum-free Protein Block (Dako,
Carpentaria, CA, USA) for $30 \mathrm{~min}$ to eliminate possible non-specific antibody binding. To determine the specificity of each primary antibody, the primary antibody in control coverslips was replaced with appropriate pre-immune serum. The primary antibodies used for cell staining include E-cadherin (1:500) and N-cadherin (1:500) from BD Biosciences; Fibronectin (1:200), vimentin (1:100), acidic Cytokeratin (1:50), and $\beta$-catenin (1:500) all from Abcam; ZO-1 (1:100) from Invitrogen; and CCN1 (1:100). For immunofluorescence, FITC- or Texas Red-conjugated secondary QJ;antibodies (Abcam) were diluted in PBS according to the manufacturer's recommendations. Nuclei were counter stained with propidium iodide (Invitrogen). Otherwise, cells were stained with a LSAB + kit (Dako) containing a biotinylated universal linker and a horseradish peroxidaseconjugated streptavidin instead, following the manufacturer's protocol. In this case, AEC Chromogen (Dako) was used as a substrate. Nuclei were counter stained with hematoxylin (Sigma-Aldrich). To show actin polymerization, cells were fixed in $3.7 \%$ formaldehyde (Sigma-Aldrich) for $10 \mathrm{~min}$, permeabilized in $0.1 \%$ Triton X-100 (Thermo Fisher Scientific, Pittsburg, PA, USA) for $5 \mathrm{~min}$, and incubated with Oregon Green 488 phalloidin (Invitrogen) for $20 \mathrm{~min}$.

\section{Wounding Assay}

Cells were cultured on collagen-coated microscopic slides till confluent. Incisions were made at $1-\mathrm{cm}$ intervals with a sterile $1000 \mu \mathrm{l}$-pipette tip following the lines drawn on the back of the slides before sterilization as described in our earlier studies. ${ }^{8,24}$ Cells were returned to culture until the denuded area was completely filled by migrating cells. During this period of recovery, two of the slides were removed every $12 \mathrm{~h}$ to process for cell staining. Wound closure was quantified under a microscope by counting the number of cells that had migrated across the starting line. Five microscopic fields were randomly selected per slide per condition to count the cells and five slides were used for each condition $(n=25)$.

\section{Experimental Gastric Wound Healing}

The animal study was approved by the Subcommittee for Animal Studies of the VA Long Beach Healthcare System. Sixty-six male Sprague-Dawley rats (Charles Rivers Labs, Wilmington, MA, USA) with body weight from 225 to $250 \mathrm{~g}$ were fasted $12 \mathrm{~h}$ before operation. The animals were anesthetized by intraperitoneal injection of pentobarbital at $50 \mathrm{mg} / \mathrm{kg}$ body weight. Gastric ulcers were induced in 60 rats by topical application of $50 \mu \mathrm{l}$ acetic acid to the serosa of glandular stomach at the anterior wall through a polyethylene tube $(\varnothing 4.0 \mathrm{~mm})$ for $90 \mathrm{~s}$ as described in our earlier studies. ${ }^{8,24}$ Six rats per time point were euthanized at $1,3,7$, and 14 days after ulcer induction to collect gastric tissue specimen.

The remaining 36 rats with stomach wounds were divided into three groups (12 rats each) and each group was given a 
mucosal injection (around the wound) of either $100 \mu \mathrm{l}$ of PBS (control), $100 \mathrm{ng}$ of recombinant CCN1 in $100 \mu \mathrm{l}$ of PBS, or $100 \mathrm{ng}$ of CCN1 mixed with $10 \mu \mathrm{g}$ of CCN1 antibody in $100 \mu \mathrm{l}$ of PBS. Six rats were sham operated as control. All rats were euthanized 7 days after injection to assess wound healing.

\section{Histology and Immunohistochemistry}

Gastric tissue was fixed in 10\% formalin overnight and embedded in paraffin. Antigen was retrieved by pepsin digestion. Tissue sections were stained with either hematoxylin/eosin for histological evaluation, or an antibody against one of the following proteins: CCN1 (1:200), vimentin (1:400), smooth muscle $\alpha$-actin (SM $\alpha$-actin, 1:100, Abcam), and GST-tag (1:500). The LSAB + kit was used to develop a signal. The effect of CCN1 treatment on wound healing was evaluated by measuring ulcer size under a dissecting microscope.

\section{Statistical Analysis}

All numerical data are expressed as mean \pm s.d. and analyzed by single classification one-way ANOVA and $P<0.05$ was considered as significant.

\section{RESULTS}

\section{Epithelial Injury Induces Concurrent Transient} Expression of CCN1 and Mesenchymal Markers

From our earlier studies, ${ }^{8,24}$ we have learned that epithelial injury (eg ulceration) activates epithelial cells adjacent to the wound to migrate toward the center of the wound to cover the denuded area. One aim of this study was to determine whether these migrating cells experience a process of EMT. To answer this question, we created experimental ulcers in rat stomach and stained gastric tissue sections at the different stages of ulcer development and healing for various mesenchymal markers (eg N-cadherin, vimentin, fibronectin). At day 3, when re-epithelialization started, many epithelial cells at the ulcer margin showed positive signals for mesenchymal markers, such as vimentin (Figure 1a, upper panel), suggesting that EMT might be taking place in these cells. Concurrently, a massive amount of CCN1 was found in the ECM of the ulcer margin (Figure 1a, lower panel), which is never seen in normal gastric mucosa (Figure 1a, left panel), suggesting a possible causal relationship between $\mathrm{CCN} 1$ and the expression of mesenchymal markers. Moreover, when these migrating epithelial cells recovered the denuded area ( $\sim 14$ days), the expression of CCN1 in the wounded epithelium was no longer detectable, and neither were the mesenchymal proteins (Figure 1a, right panel), suggesting that injury-activated EMT might be reversible.

To validate this finding, we mimicked this event in cultured rat gastric epithelial cells-RGM1. We created wounds in the confluent RGM1 cell monolayer by scratching it with a pipette tip. Cells were returned to regular culture medium to grow till the incisions were completely refilled by migrating cells from two sides. During this period of healing, cells were removed from culture every $12 \mathrm{~h}$ and stained for the same antigens that were examined in tissue sections. Consistent with our in vivo observation, cells at the wound margin showed a distinct expression of mesenchymal markers such as vimentin (Figure 1b, left panel), which are not characteristics of normal RGM1 cells, indicating occurrence of a mesenchymal transition. However, after the incisions were completely healed ( $\sim 4$ days), these features disappeared (Figure 1b). CCN1 expression showed similar dynamics to vimentin (Figure $1 \mathrm{~b}$, right panel). However, when similar experiments were done in CCN1-deficient RGM1 cells $(\mathrm{CCN}-)$, the expression of mesenchymal markers at the wound margin was dramatically reduced (Figure 1c) and the healing process was also slowed down by $71 \pm 5 \%(P<0.01)$ compared with the negative shRNA treatment, supporting our hypothesis that injury-induced $\mathrm{CCN} 1$ expression in epithelial cells mediates a transient expression of mesenchymal proteins in these cells, which is critical for proper wound healing.

\section{CCN1 Induces EMT In Vitro}

Expression of mesenchymal markers in epithelial cells is a sign of EMT. ${ }^{1,2}$ To confirm that CCN1 can induce EMT, RGM1 cells were treated with a recombinant human CCN1 protein at $100 \mathrm{ng} / \mathrm{ml}$ for $24 \mathrm{~h}$. RNA was collected from the cells and examined for expression of an extended list of mesenchymal genes including VIM (coding for vimentin), S100A4, MMP9, CDH2 (coding for N-cadherin), FN1 (coding for fibronectin), POSTN (coding for periostin), and FGF2 (coding for basic fibroblast growth factor), as well as a panel of epithelial markers such as $\mathrm{CDH} 1$ (coding for E-cadherin), CTNNB1 (coding for $\beta$-catenin), OCLN (coding for occludin), TJP1 (coding for ZO-1), DSG1 (coding for desmoglein), KRT14 (coding for cytokeratin-14), and MUC1 (coding for mucin-1). Rat2 fibroblasts were included in the experiment as a positive control for mesenchymal markers. Under CCN1 treatment, all of the mesenchymal genes tested, especially VIM, MMP9, and CDH2, were significantly upregulated (Figure 2a). CDH2 level in the CCN1-treated RGM1 cells was even higher than it was in Rat2 fibroblasts. On the other hand, all of the epithelial markers, except $\mathrm{CDH} 1$ and CTNNB1, showed a dramatic reduction in expression in the presence of CCN1 (Figure 2b). We also stained the cells for expression of these markers at the protein level. CCN1 did not only activate mesenchymal protein expression but also induced tremendous actin polymerization (Figure 2c), indicating an increase in cell mobility. For the epithelial markers, keratin expression was mostly lost in the presence of CCN1, whereas the components of epithelial adherens junctions (eg E-cadherin, $\beta$-catenin), tight junctions (eg occludin, ZO-1), and desmosomes (eg desmoglein, $\gamma$-catenin) were absent from their normal membrane location and appeared in the cytoplasm or the nucleus (Figure 2d). 
These results were also observed in the RGM1 cells transfected with CCN1 cDNA plasmid (data not shown). Taken together, these data confirmed that CCN1 can induce EMT.

\section{CCN1 Induces E-Cadherin Ubiquitination}

On the basis of the RT-PCR results, CCN1 treatment did not significantly alter the expression of $\mathrm{CDH} 1$ and CTNNB1, a

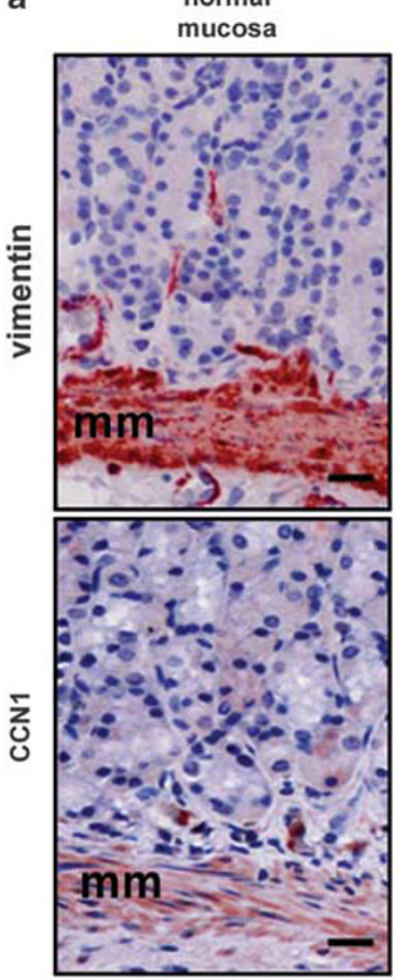

b

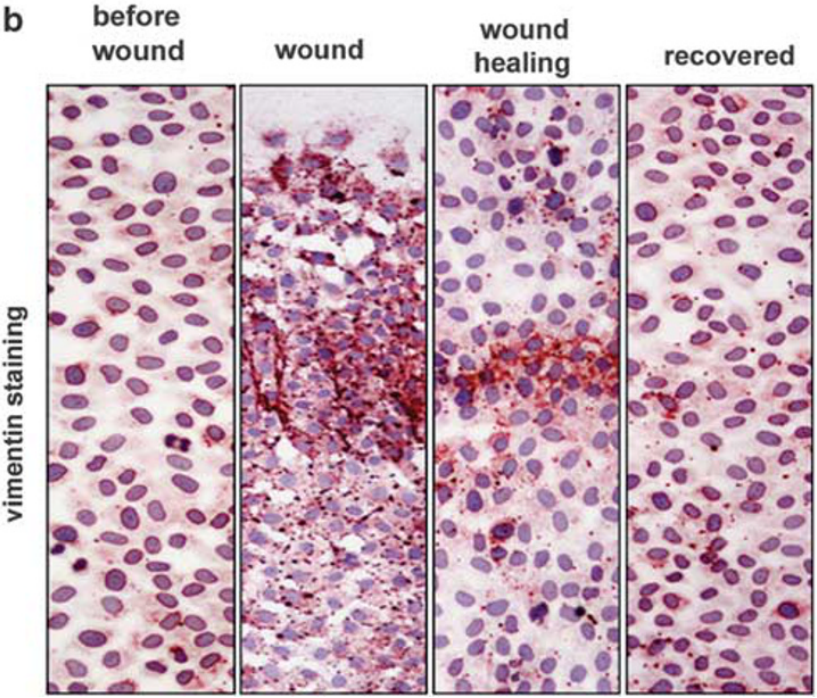

ulcer margin (day 3 )
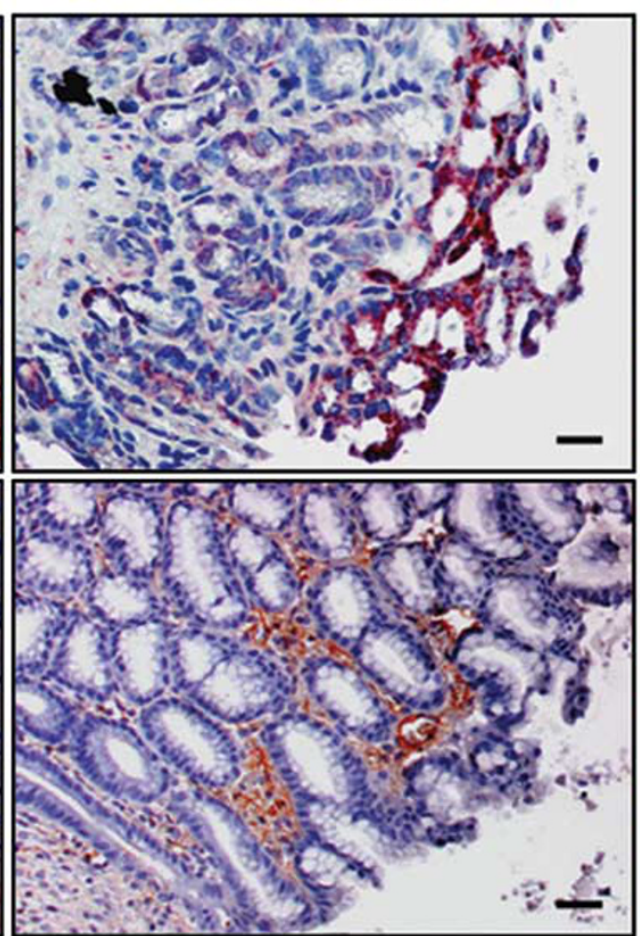

re-epithelialization (day 14)


\section{before

wound


Figure 1 Epithelial injury induces concurrent transient expression of CCN1 and vimentin. Gastric ulcers or epithelial injury was induced as described in Materials and methods. Nuclei were counter stained by hematoxylin. (a) Gastric ulcers were induced in rats and thereafter examined at 1, 3, 7, and 14 days


epithelial cells at day 3 showed strong vimentin expression (middle in upper panel). Meantime, accumulation of CCN1 was found in the ECM of such a location (middle in lower panel). Both of these molecules vanished after the wounded mucosa was re-epithelialized at day 14 (right). mm, muscularis mucosa. Scale bar, $10 \mu \mathrm{m}$. (b) RGM1 cells were cultured on collagen-coated microscopic slides to form a monolayer and wounded by scratching with a pipette tip. Cells were then stained for vimentin and CCN1 every $12 \mathrm{~h}$ till the denuded area was fully recovered by migrating cells. RGM1 cells at wound

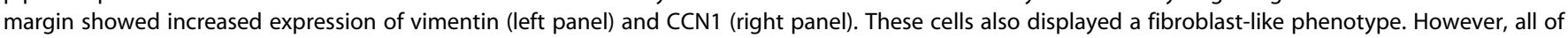
these features disappeared after the re-epithelialization process was completely finished. (c) CCN1 was knocked down by specific shRNA transfection in

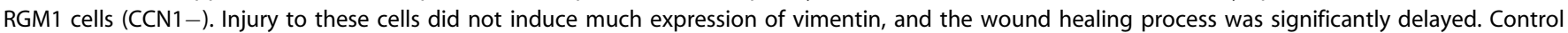
cells were transfected with a negative shRNA (control). 
C

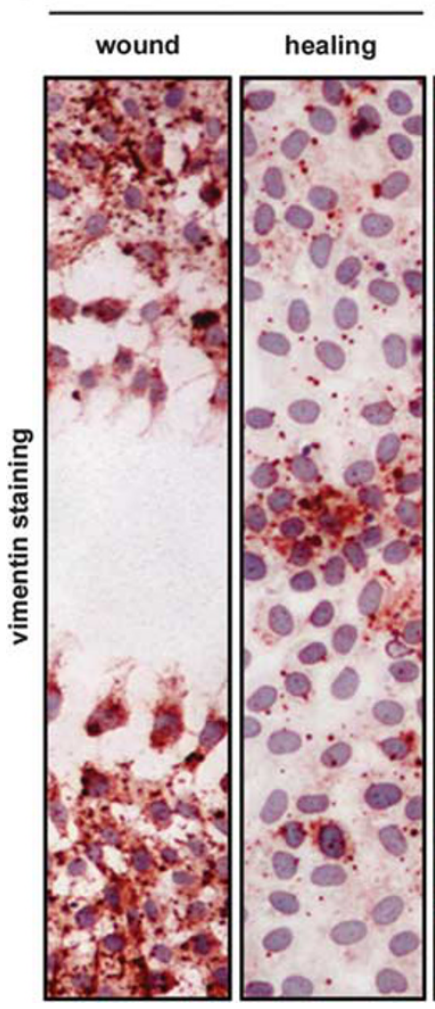

CCN1-

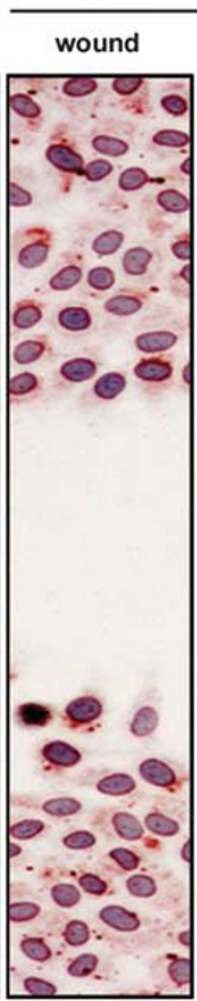

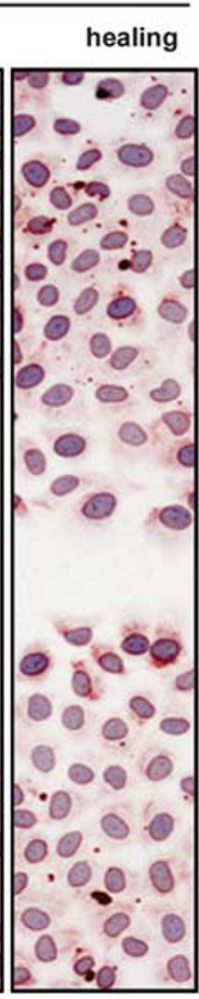

Figure 1 Continued.

whereas cell staining showed a signal reduction (Figure 2b). To determine whether CCN1 causes E-cadherin loss, we examined E-cadherin expression by western blotting. As the CCN1 treatment extended, E-cadherin levels gradually declined (Figure 3a). At day 4, only $30 \%$ of E-cadherin remained (Figure 3b). In the western blot, E-cadherin antibody also picked up multiple bands in the lanes of samples collected after extended CCN1 treatment (Figure 3a), indicating possible E-cadherin degradation. To confirm whether E-cadherin was ubiquitinated in the presence of CCN1, which would lead to its degradation, we pulled down E-cadherin and probed with an antibody against Ubiquitin. Extensive ubiquitination of E-cadherin was observed after 4 days of $\mathrm{CCN} 1$ treatment (Figure 3c). On the basis of the literature, E-cadherin loss in epithelial cells can also result from transcriptional inhibition through activation of its negative regulators, for example Snail, ${ }^{19}$ Slug, ${ }^{25}$ or Twist. ${ }^{26} \mathrm{To}$ determine whether these transcription factors are also involved in CCN1-induced E-cadherin loss, we examined the effect of CCN1 on their expression. As shown in Figure $3 \mathrm{~d}$ and e, CCN1 treatment did not significantly alter these transcription factors at either mRNA or protein level, consistent with the result shown in Figure $2 \mathrm{~b}$.

CCN1 Induces Reversible $\beta$-Catenin Activation through ILK $\beta$-catenin is a molecule with a dual function. ${ }^{18}$ Serine-37 phosphorylated $\beta$-catenin serves as a component of epithelial

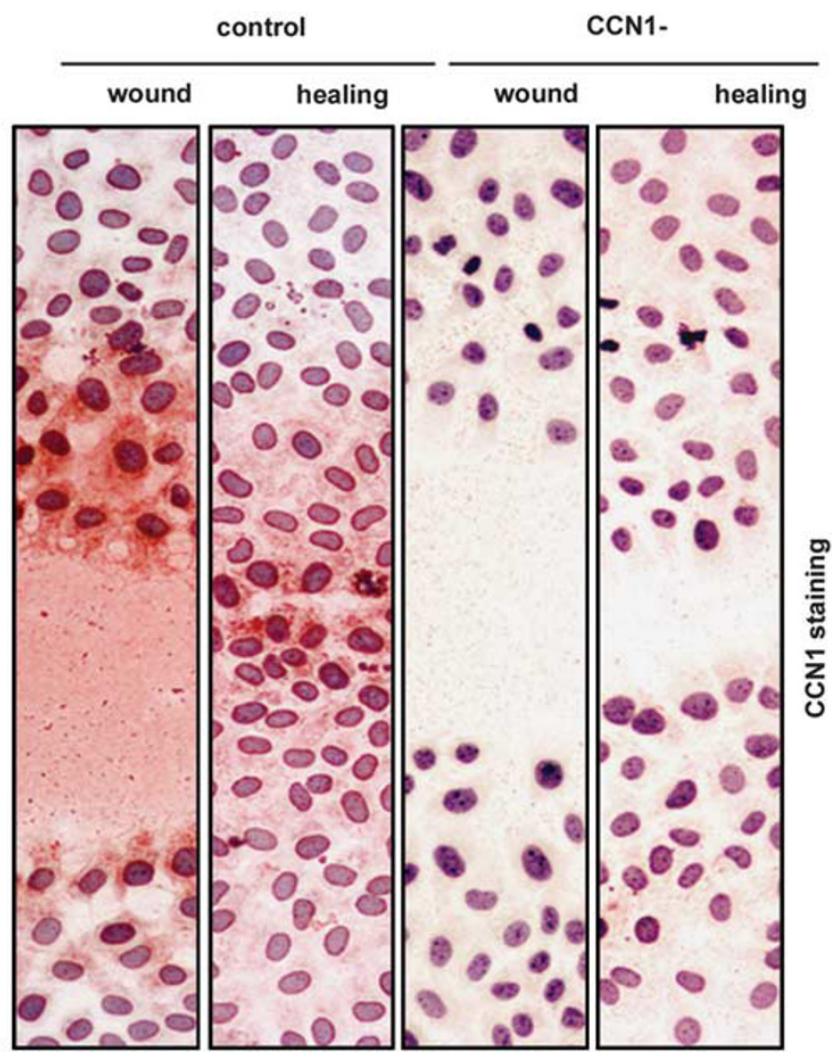

adherens junctions, and excess cytoplasmic phospho$\beta$-catenin is targeted for degradation. However, when it is dephosphorylated, $\beta$-catenin dissociates from E-cadherin, is stabilized and allowed to accumulate in the cytoplasm, and subsequently translocates to the nucleus, where it becomes a transcriptional co-activator in complex with LEF-1. It has been reported that $\beta$-catenin dissociation from the cell membrane can cause E-cadherin ubiquitination. ${ }^{27}$ To determine whether this is the mechanism for CCN1-induced E-cadherin degradation, we examined the effect of CCN1 on $\beta$-catenin in RGM1 cells. From western blot analyses, $\beta$-catenin became activated (Serine-37 dephosphorylation) in the presence of CCN1 (Figure 4a). However, when the cells were cultured with CCN1 for 4 days and then replated in regular medium (without addition of $\mathrm{CCN} 1$ ), $\beta$-catenin could become phosphorylated again (Figure 4a, recovery), suggesting that $\mathrm{CCN} 1$-induced $\beta$-catenin activation is reversible. Immunoprecipitation assays showed that $\beta$-catenin became associated with LEF-1 in the presence of $\mathrm{CCN} 1$ (Figure $4 \mathrm{~b}$ ), confirming $\beta$-catenin activation. Consistent with the immunoprecipitation/western blot results, immunofluorescence microscopy clearly showed that $\beta$ catenin disappeared from its primary membrane localization and accumulated in the nucleus along with LEF-1 under $\mathrm{CCN} 1$ treatment (Figure 4c), whereas, after recovery in regular medium for $48 \mathrm{~h}, \beta$-catenin resumed its membrane association. 




b

RGM1 $\|$ RGM1/CCN1 $=$ Rat2

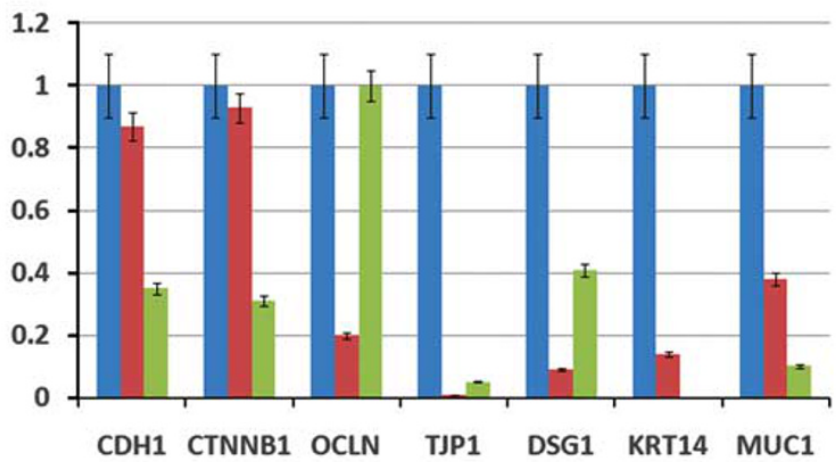

C

RGM1


RGM1/CCN1


Rat2
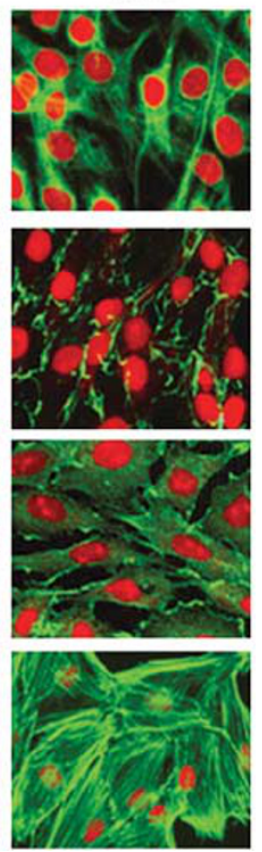

d
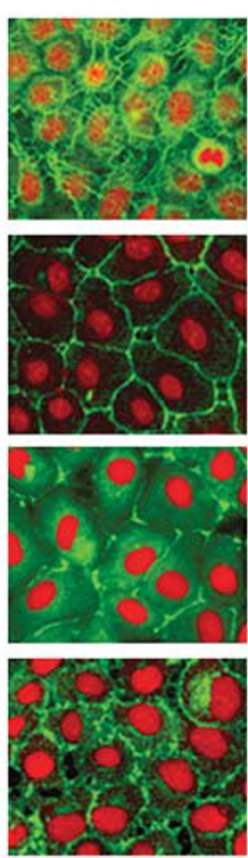

RGM1/CCN1


Rat 2


Figure 2 CCN1 induces EMT in RGM1 cells. Normal rat gastric mucosal epithelial RGM1 cells were compared with rat fibroblast Rat2 cells (positive control for mesenchymal markers). (a) Real-time RT-PCR shows that 24-h treatment with recombinant CCN1 protein induces expression of mesenchymal markers including VIM, S100A4, MMP9, CDH2, FN1, POSTN, and FGF2 in RGM1 cells. Values presented in the graphs are fold changes compared with RGM1 baseline. (b) Real-time RT-PCR shows that 24-h treatment with recombinant CCN1 protein suppresses expression of epithelial markers including CDH1, CTNNB1, OCLN, TJP1, DSG1, KRT14, and MUC1 in RGM1 cells. Values presented in the graph are fold changes compared with RGM1 baseline. (c) Cell staining shows that 48-h treatment with recombinant CCN1 protein activates expression of mesenchymal proteins such as vimentin, fibronectin, and N-cadherin and increases actin polymerization in RGM1 cells. (d) Cell staining shows that 48-h treatment with recombinant CCN1 protein deprives epithelial junctional

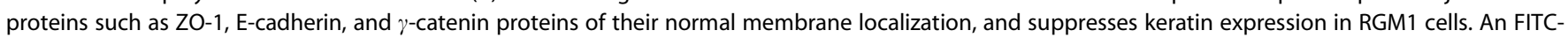
conjugated secondary antibody was used to develop the signals. The nucleus was counter stained with propidium iodine. F-actin was identified with Oregon Green 488-conjugated phalloidin.

All of the known cellular activities of CCN1 are mediated through a pool of integrin receptors. ${ }^{14}$ As an integrin-associated protein, ILK negatively regulates GSK-3 $\beta$, which stabilizes adherens junctions by keeping $\beta$-catenin phosphorylated at serine-37. Overexpression of ILK in tubular epithelial cells was found to dissociate $\beta$-catenin from E-cadherin. ${ }^{16}$ To determine whether ILK is responsible for $\mathrm{CCN} 1$-induced dissociation of E-cadherin and $\beta$-catenin in RGM1 cells, we first measured ILK expression and kinase activity (using recombinant GSK-3 $\beta$ as a substrate) in response to CCN1 treatment. CCN1 did not affect ILK expression levels significantly, but it dramatically increased ILK activity by $147 \pm 18 \%(P<0.001)$ at $24 \mathrm{~h}$ (Figure $4 \mathrm{~d})$, similar to the effect of hyperactive ILK expression (ILK +), which was used as positive control for ILK activity. Next, we sought to test whether CCN1-induced $\beta$-catenin activation is ILK dependent. We examined GSK-3 $\beta$ activity using recombinant $\beta$-catenin as a substrate and found that CCN1 inhibits GSK-3 $\beta$ activity. However, when we knocked ILK down with shRNA (ILK-), CCN1 failed to inactivate GSK-3 $\beta$ 
a
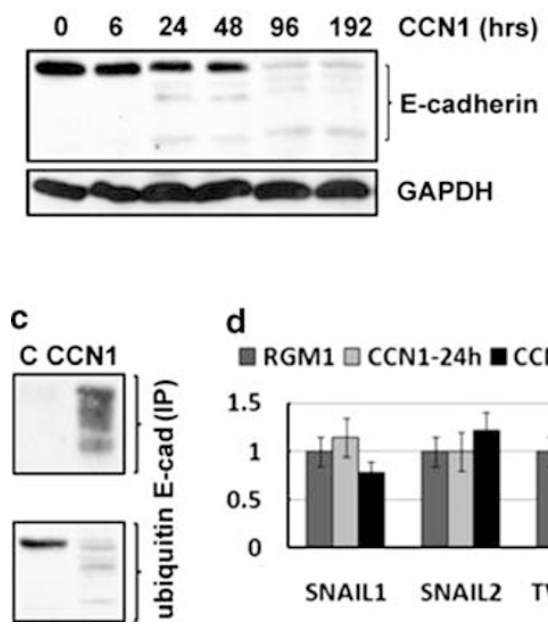

\section{d}

$\square$ RGM1 $\square$ CCN1-24h $\mathrm{CCCN1}-48 \mathrm{~h}$

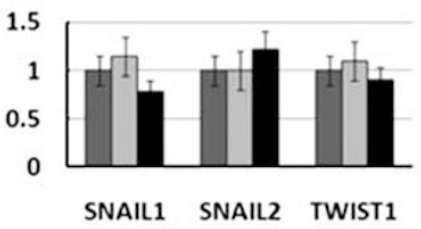

b

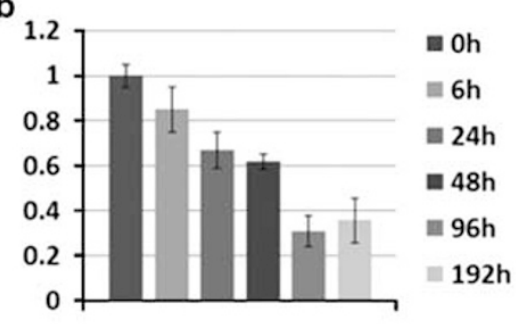

e
0

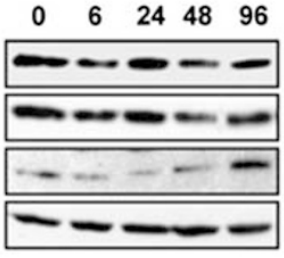

CCN1 (hrs)

Snail

Slug

Twist

GAPDH

Figure 3 Cyr61 induces E-cadherin degradation through ubiquitination. (a) Western blot analysis shows that E-cadherin levels progressively decline in RGM1 cells with CCN1 treatment. A degradation ladder can be seen after 24-h treatment with CCN1. GAPDH was used as a loading control.

(b) Quantification of E-cadherin loss under CCN1 treatment. (c) Immunoprecipitation of E-cadherin shows that 96-h CCN1 treatment causes E-cadherin degradation (lower) in association with Ubiquitin (upper). (d) Real-time RT-PCR shows that CCN1 treatment does not significantly alter the expression of E-cadherin transcriptional inhibitors including SNAIL1, SNAIL2 (Slug), and TWIST1 at the mRNA level in RGM1 cells. (e) Western blot analysis shows that protein expression of Snail, Slug, and Twist are not affected by CCN1 treatment. GAPDH was used as a loading control.

(Figure 4e), indicating that ILK mediates CCN1-induced $\beta$-catenin dephosphorylation. Expression of hyperactive ILK had a similar effect as CCN1 treatment, further supporting our conclusions.

\section{CCN1 Promotes Re-Epithelialization In Vivo during Ulcer Healing}

On the basis of the results obtained above, we sought to test whether in vivo utilization of CCN1 has a similar effect on epithelial cells. We created gastric ulcers in 36 rats. After ulcer induction, each group of 12 rats was given a mucosal injection (around the wound) of either PBS (control), recombinant $\mathrm{CCN} 1(\mathrm{CCN} 1+)$, or $\mathrm{CCN} 1$ mixed with excessive neutralizing antibody $(\mathrm{CCN} 1+\mathrm{Ab})$. An additional six rats were sham operated as control. All of the rats were euthanized 7 days later. CCN1 expression in gastric mucosa was mainly found near the wounds (Figure 5a, first row) where some epithelial cells showed positive staining for vimentin and SM $\alpha$-actin (Figure 5a, third and fourth rows). However, injection of recombinant CCN1 protein, as identified by GST-tag (Figure 5a, second row), induced a lot more cells to express vimentin (Figure 5a, third row) and some of them also expressed SM $\alpha$-actin (Figure 5a, fourth row), indicative of a myofibroblast phenotype (indicated by arrows). Similar staining in response to CCN1 treatment was also observed when CCN1 was injected into normal mucosa $\sim 5 \mathrm{~mm}$ away from the edge of the wound, where CCN1 expression was unlikely activated (Figure 5a, left column), confirming that exogenous CCN1 expression is capable of inducing the observed increase in vimentin and SM $\alpha$-actin. The effect of the different treatments on $\mathrm{CCN} 1$ protein expression levels was also confirmed by western blot analysis (data not shown). Ulcer closure is a combinational effort of regional epithelial migration/proliferation and myofibroblast contraction. CCN1 injection significantly reduced ulcer size by $73 \pm 12 \%$, and CCN1 neutralization delayed ulcer closure, as reflected by $110 \pm 25 \%$ larger size compared with PBS injection (both $P<0.01$, Figure $5 \mathrm{~b}$ and c), demonstrating that CCN1 can induce EMT in vivo and promote re-epithelialization during wound healing.

\section{DISCUSSION}

In the last decade, a rapidly increasing number of studies have recognized $\mathrm{CCN}$ proteins as major players in global control over most, if not all, cellular activities in organ development and tissue regeneration. ${ }^{3,4}$ Because of their heparin affinity, these matricellular molecules fill the functional and physiological gap between structural molecules in the ECM and soluble molecules like growth factors. Although the importance of growth factors in ulcer healing has been intensively investigated, the role of CCN members in this process is still a mystery.

CCN1 is known for supporting cell adhesion, proliferation, migration, differentiation, and angiogenesis-the fundamental biological processes required for wound healing. However, our knowledge about CCN1 thus far has been mostly gained through studies on either fibroblasts or cancers. $^{28,29}$ Its effect on normal epithelial cells is largely unknown. In this study, we have revealed several new characteristics of CCN1 in gastric epithelial cells and tissue, including activation by injury, induction of mesenchymal markers in epithelial cells, enhancement of $\beta$-catenin stability 




c

RGM1
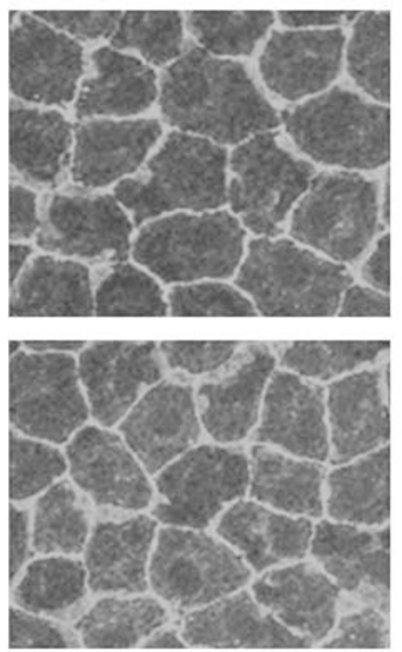

CCN1-24 hrs
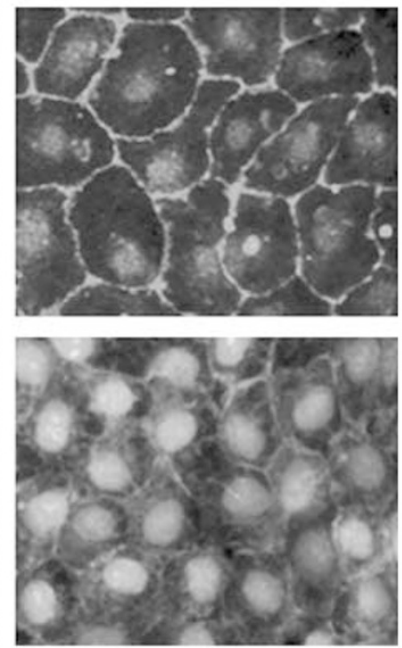

d

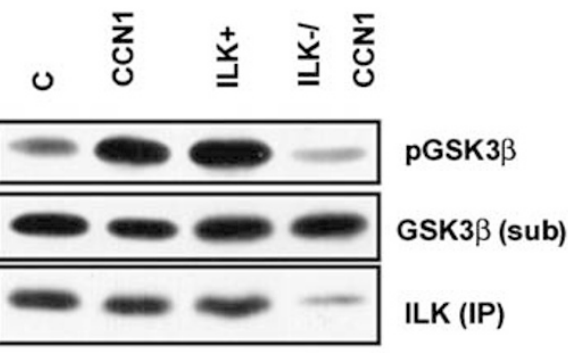

b C CCN1



CCN1-96 hrs/ recovery- $48 \mathrm{hrs}$
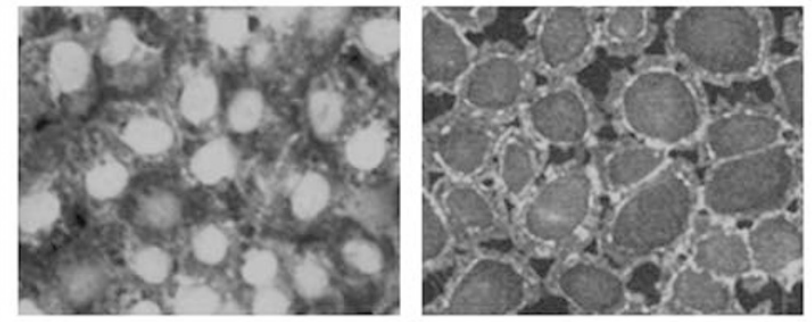

志
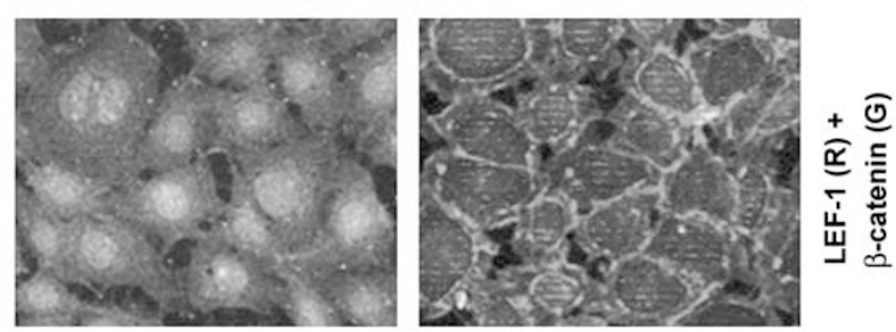

e

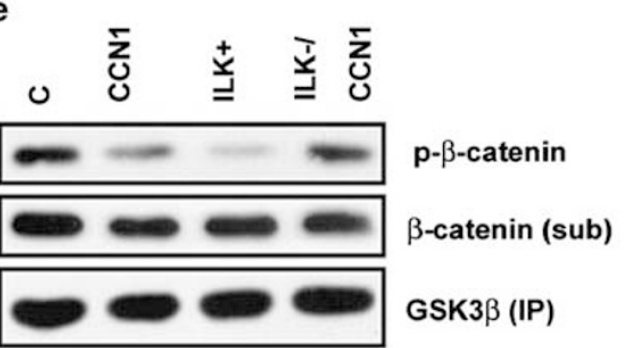

Figure 4 CCN1 induces reversible $\beta$-catenin activation through ILK. (a) Western blot analysis shows that CCN1 treatment activates $\beta$-catenin, and that this effect can be reversed through recovery in regular medium. GAPDH was used as a loading control. (b) Immunoprecipitation of $\beta$-catenin shows that activation of $\beta$-catenin, in response to CCN1 treatment, is associated with LEF-1. (c) Immunofluorescence microscopy shows that $\beta$-catenin becomes nuclear localized under CCN1 treatment in RGM1 cells (upper panel); however, when the cells are allowed to recover in regular growth medium for $48 \mathrm{~h}$, membrane association of $\beta$-catenin can be restored. The lower panel, double stained for $\beta$-catenin (green) and LEF-1 (red), shows that $\beta$-catenin and LEF-1 are colocalized (yellow) in the nucleus under the influence of CCN1. (d) ILK kinase assay using recombinant GSK-3 $\beta$ as a substrate shows that CCN1 treatment upregulates ILK activity, as expression of hyperactive ILK (ILK + ) does. Knockdown of ILK (ILK-) with shRNA prevents this action. (e) GSK-3 $\beta$ kinase assay using recombinant $\beta$-catenin as a substrate shows that CCN1 treatment inhibits GSK-3 $\beta$ activity as reflected by the amount of ser-37-phosphorylated $\beta$-catenin, but knockdown of ILK can prevent this action.

and E-cadherin degradation, activation without induction of ILK, and promotion of re-epithelialization during wound healing.

E-cadherin is the main cell-cell adhesion molecule in the epithelium and is generally regarded as a master organizer of the epithelial phenotype. ${ }^{30}$ Loss of E-cadherin during EMT has been largely attributed to either its gene mutations or transcriptional repression. ${ }^{31-33}$ However, several studies observed a loss of E-cadherin in different tissues despite its normal mRNA expression. ${ }^{34}$ The observed CCN1-induced E-cadherin decline in gastric cells falls in this category. Under the influence of CCN1, E-cadherin degrades rapidly by ubiquitination, whereas its mRNA level remains unchanged. Whether $\beta$-catenin has a role in E-cadherin stabilization is 
a



b



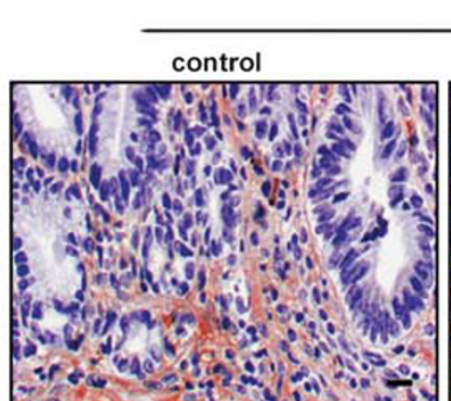

wounded mucosa
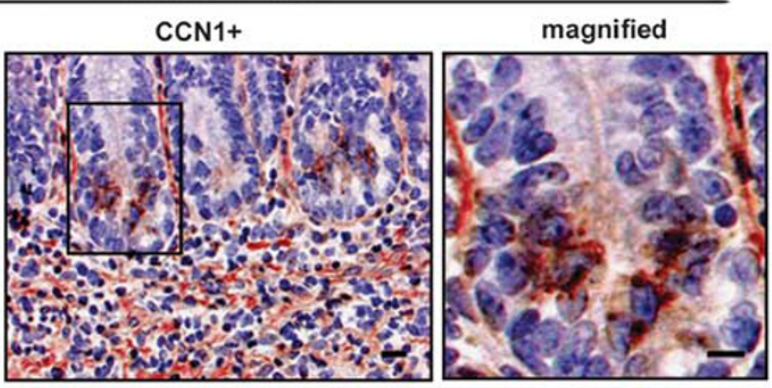

¿্
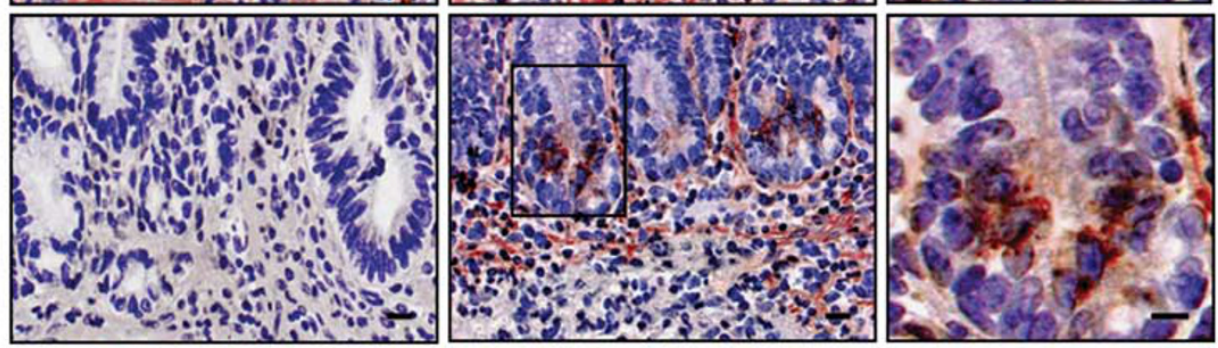

占
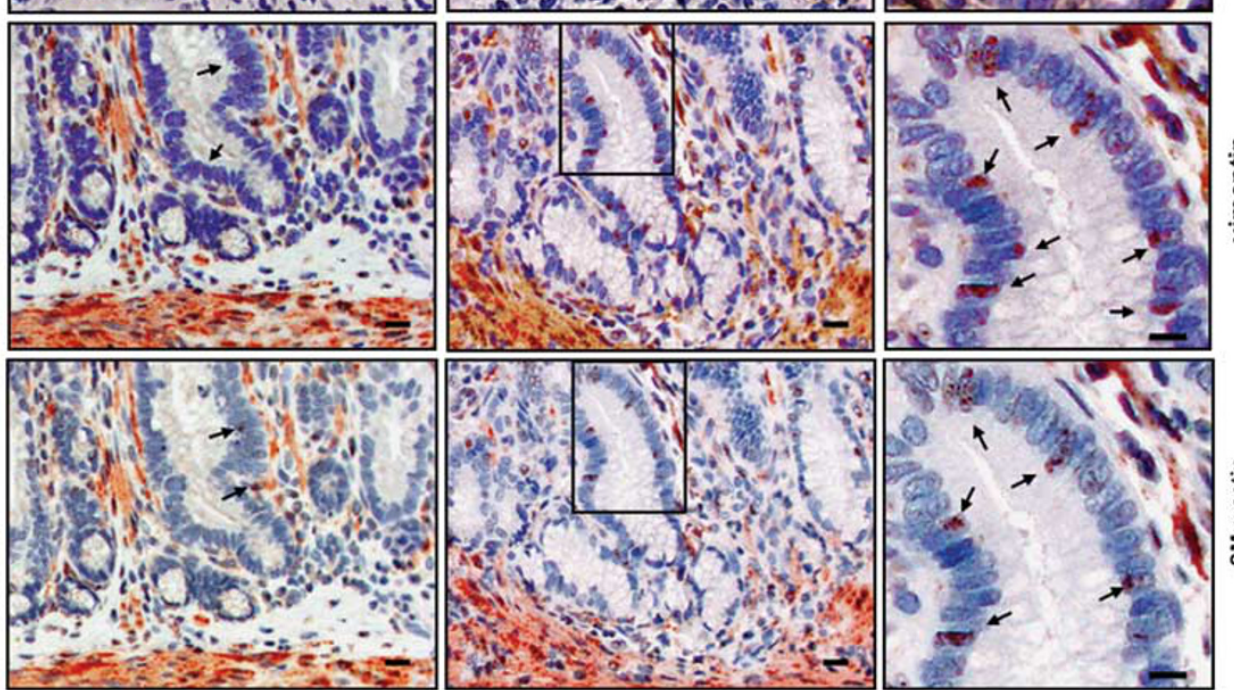

总

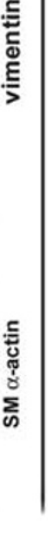

C

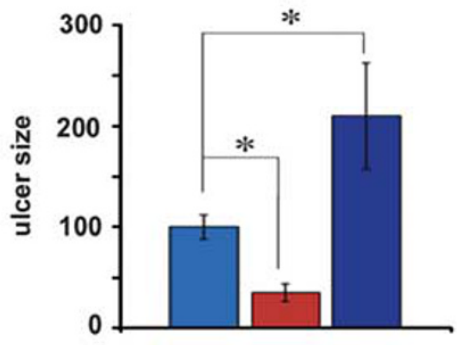

control $\square \mathrm{CCN} 1+\square \mathrm{CCN} 1+\mathrm{Ab}$

Figure 5 Injection of recombinant CCN1 protein into gastric mucosa induces vimentin and SM $\alpha$-actin expression and promotes re-epithelialization during ulcer healing. After inducing gastric ulcers in rats, rats received mucosal injection of either PBS (control), recombinant CCN1 (CCN1 + ), or recombinant CCN1 mixed with excessive neutralizing antibody $(C C N 1+A b)$ around the ulcer induction site. Rats were euthanized 7 days after treatment. (a) Tissues from normal mucosa injected either with vehicle (control) or with recombinant CCN1 (CCN1 +) were compared with tissues from wounded mucosa with the same treatment. CCN1 + samples from wounded mucosa were also magnified for better visualization (right-most column). The first row identifies the location of CCN1 (both endogenous and exogenous) under each condition by immune staining for CCN1, showing that CCN1 is upregulated in regenerating mucosa in both control and CCN1 + samples of wounded tissue compared with normal mucosa control. The second row specifically identifies the exogenous CCN1 by immune staining for GST-tag, confirming the locations of injection. The third row shows vimentin expression in mucosal epithelial cells (arrows) where CCN1 is found. The fourth row indicates that some of the vimentin-positive cells are myofibroblasts (arrows). Scale bar, $10 \mu \mathrm{M}$. (b) Stomach dissection shows improved ulcer healing by CCN1 treatment (CCN1 +) and delayed ulcer closure due to neutralization of CCN1 (CCN1 + Ab).

(c) Quantification of relative ulcer size (the control was set to $100 \%)$. ${ }^{*}$ indicates statistical significance $(P<0.01)$. 
still debatable. It was proposed that there might be two separate pools of $\beta$-catenin existing in epithelial cells and that the cadherin-associated $\beta$-catenin might never become available for transcriptional signaling. ${ }^{35}$ In support of this theory, two different $\beta$-catenin homologs were found in Caenorhabditis elegans. ${ }^{36}$ In addition, removing $\beta$-catenin in endothelial cells had no effect on VE-cadherin stability. ${ }^{37}$ Conversely, biochemical examination shows that E-cadherin contains a high degree of PEST sequences (motifs associated with Ubiquitin/proteosome degradation) within its $\beta$-catenin-binding region; therefore, E-cadherin is unstructured and targeted for degradation when not bound to $\beta$-catenin. ${ }^{27}$ Our study showed that CCN1 induces $\beta$-catenin nuclear translocation and E-cadherin ubiquitination in RGM1 cells, supporting a critical role of $\beta$-catenin association in protecting E-cadherin stability.

Vimentin is a type III intermediate filament protein exclusively expressed in mesenchymal cells. However, several studies described transient activation of vimentin expression in epithelial cells under certain pathological or physiological conditions that require epithelial cell migration. ${ }^{38,39}$ For this reason, vimentin has been used to symbolize the migratory status of epithelial cells. A putative $\beta$-catenin-binding site was recently identified in the VIM gene promoter, and mutations in this region impair vimentin transcriptional activation in breast cancer cells. ${ }^{40}$ Given the positive role of CCN1 in inducing $\beta$-catenin nuclear translocation and association with LEF-1, the presence of a putative $\beta$-catenin-binding site in the VIM gene promoter might explain how CCN1 induces vimentin expression in RGM1 cells, confirming VIM as a $\beta$-catenin target gene.

Communications between cells and the ECM are mediated by integrins and a complex network of proteins that link integrins to the cytoskeleton. ILK is one of these proteins. Although ILK was discovered only a decade ago, ${ }^{15}$ an increasing amount of data links it to several important cellular activities including EMT. ${ }^{16}$ Our recent study found that ILK serves as a mediator in SRF phosphorylation and transcriptional activation; for this reason, dysfunction of ILK impairs TGF $\beta 1$-induced EMT in esophageal epithelial cells. ${ }^{9}$ As an SRF target, CCN1 is shown in our current study to increase ILK kinase activity in gastric epithelial cells and knockdown of ILK prevents CCN1-induced $\beta$-catenin activation. This CCN1-ILK-SRF-CCN1 loop might serve as a mechanism to amplify the signal for wound healing.

In this study, we showed both in vitro and in vivo that injury-induced CCN1 is critical for promoting mesenchymal phenotype in gastric epithelial cells, which facilitated them to move into the wound to restore epithelial continuity. Most important, we found this effect reversible. Once injuryinduced $\mathrm{CCN} 1$ expression is reduced back to normal on wound closure, those transformed cells regain normal epithelial phenotype. This makes CCN1 a more favorable molecular candidate for the development of new treatment strategies in wound healing, because EMT not only occurs during wound healing, but is also involved in the development of malignancies and their metastatic potential. Inappropriate and sustained/irreversible induction of EMT can have disastrous consequences, such as visceral fibrosis and the development of malignancies. Therefore, although more detailed studies would be required to optimize the ideal window of CCN1 levels to ensure effective wound closure without aberrant transformation, the transient feature of CCN1-induced EMT is of great value for its future potential in clinical applications. Moreover, due to the great similarities in molecular dynamics between wound healing and cancer progression, the findings from this study may also benefit the processes for diagnosing, characterizing, and the treatment regimens for certain malignancies.

\section{ACKNOWLEDGEMENT}

This study was supported by the Department of Veterans Affairs of the United States and the American Heart Association grants to Dr Chai.

\section{DISCLOSURE/CONFLICT OF INTEREST}

The authors declare no conflict of interest.

1. Lee JM, Dedhar S, Kalluri $\mathrm{R}$, et al. The epithelial-mesenchymal transition: new insights in signaling, development, and disease. J Cell Biol 2006;172:973-981.

2. Radisky DC. Epithelial-mesenchymal transition. J Cell Sci 2005;118: 4325-4326.

3. Brigstock DR. The CCN family: a new stimulus package. J Endocrinol 2003;178:169-175.

4. Perbal B. CCN proteins: multifunctional signalling regulators. Lancet 2004;363:62-64.

5. Bork $P$. The modular architecture of a new family of growth regulators related to connective tissue growth factor. FEBS Lett 1993;327: 125-130.

6. Lau LF, Nathans D. Identification of a set of genes expressed during the G0/G1 transition of cultured mouse cells. EMBO J 1985;4:3145-3151.

7. Sun Q, Chen G, Streb JW, et al. Defining the mammalian CArGome. Genome Res 2006;16:197-207.

8. Chai J, Baatar D, Tarnawski A. Serum response factor promotes re-epithelialization and muscular structure restoration during gastric ulcer healing. Gastroenterology 2004;126:1809-1818.

9. Chai J, Norng M, Tarnawski AS, et al. A critical role of serum response factor in myofibroblast differentiation during experimental oesophageal ulcer healing in rat. Gut 2007;56:621-630.

10. Chai J, Jones MK, Tarnawski AS. Serum response factor is a critical requirement for VEGF signaling in endothelial cells and VEGF-induced angiogenesis. FASEB J 2004;18:1264-1266.

11. Latinkic BV, Mo FE, Greenspan JA, et al. Promoter function of the angiogenic inducer Cyr61gene in transgenic mice: tissue specificity, inducibility during wound healing, and role of the serum response element. Endocrinology 2001;142:2549-2557.

12. Leu SJ, Chen N, Chen CC, et al. Targeted mutagenesis of the angiogenic protein CCN1 (CYR61). Selective inactivation of integrin alpha6beta1-heparan sulfate proteoglycan coreceptor-mediated cellular functions. J Biol Chem 2004;279:44177-44187.

13. Chen CC, Mo FE, Lau LF. The angiogenic factor Cyr61 activates a genetic program for wound healing in human skin fibroblasts. J Biol Chem 2001;276:47329-47337.

14. Lau LF, Lam SC. The CCN family of angiogenic regulators: the integrin connection. Exp Cell Res 1999;248:44-57.

15. Hannigan GE, Leung-Hagesteijn C, Fitz-Gibbon L, et al. Regulation of cell adhesion and anchorage-dependent growth by a new beta 1-integrin-linked protein kinase. Nature 1996;379:91-96.

16. Li Y, Yang J, Dai C, et al. Role for integrin-linked kinase in mediating tubular epithelial to mesenchymal transition and renal interstitial fibrogenesis. J Clin Invest 2003;112:503-516. 
17. Doble BW, Woodgett JR. GSK-3: tricks of the trade for a multi-tasking kinase. J Cell Sci 2003;116:1175-1186.

18. Liu C, Li Y, Semenov M, et al. Control of beta-catenin phosphorylation/ degradation by a dual-kinase mechanism. Cell 2002;108:837-847.

19. Zhou BP, Deng J, Xia W, et al. Dual regulation of Snail by GSK-3betamediated phosphorylation in control of epithelial-mesenchymal transition. Nat Cell Biol 2004;6:931-940.

20. Xie D, Yin D, Tong $X$, et al. Cyr61 is overexpressed in gliomas and involved in integrin-linked kinase-mediated Akt and beta-catenin-TCF/ Lef signaling pathways. Cancer Res 2004;64:1987-1996.

21. Modak C, Mouazzen W, Narvaez R, et al. CCN1 is critical for acidinduced esophageal epithelial cell transformation. Biochem Biophys Res Commun 2010;392:533-537.

22. Chen $\mathrm{Y}, \mathrm{Du} \mathrm{XY}$. Functional properties and intracellular signaling of CCN1/Cyr61. J Cell Biochem 2007;100:1337-1345.

23. Chow J, Norng M, Zhang J, et al. TRPV6 mediates capsaicin-induced apoptosis in gastric cancer cells-mechanisms behind a possible new 'hot' cancer treatment. Biochim Biophys Acta-Mol Cell Res 2007;1773:565-576

24. Nguyen T, Chai J, Li A, et al. Novel roles of local insulin-like growth factor- 1 activation in gastric ulcer healing: promotes actin polymerization, cell proliferation, re-epithelialization, and induces Cyclooxygenase-2 in a phosphatidylinositol-3 kinase dependent manner. Am J Pathol 2007;170:1219-1228.

25. Hayashida Y, Urata Y, Muroi E, et al. Calreticulin represses E-cadherin gene expression in Madin-Darby canine kidney cells via Slug. J Biol Chem 2006;281:32469-32484.

26. Kang Y, Massague J. Epithelial-mesenchymal transitions: twist in development and metastasis. Cell 2004;118:277-279.

27. Huber AH, Stewart DB, Laurents DV, et al. The cadherin cytoplasmic domain is unstructured in the absence of beta-catenin. A possible mechanism for regulating cadherin turnover. J Biol Chem 2001;276:12301-12309.
28. Bleau AM, Planque N, Perbal B. CCN proteins and cancer: two to tango. Front Biosci 2005;10:998-1009.

29. Leask A, Abraham DJ. All in the CCN family: essential matricellular signaling modulators emerge from the bunker. J Cell Sci 2006;119:4803-4810.

30. Takeichi M. Morphogenetic roles of classic cadherins. Curr Opin Cell Biol 1995;7:619-627.

31. Thiery JP. Epithelial-mesenchymal transitions in tumour progression. Nat Rev Cancer 2002;2:442-454.

32. Thiery JP. Epithelial-mesenchymal transitions in development and pathologies. Curr Opin Cell Biol 2003;15:740-746.

33. Thiery JP, Sleeman JP. Complex networks orchestrate epithelialmesenchymal transitions. Nat Rev Mol Cell Biol 2006;7:131-142.

34. D'Souza-Schorey C. Disassembling adherens junctions: breaking up is hard to do. Trends Cell Biol 2005;15:19-26.

35. Clevers H. Wnt/beta-catenin signaling in development and disease. Cell 2006;127:469-480.

36. Korswagen HC, Herman MA, Clevers HC. Distinct beta-catenins mediate adhesion and signalling functions in C. elegans. Nature 2000;406:527-532.

37. Xiao K, Allison DF, Buckley KM, et al. Cellular levels of p120 catenin function as a set point for cadherin expression levels in microvascular endothelial cells. J Cell Biol 2003;163:535-545.

38. Buisson AC, Gilles $C$, Polette $M$, et al. Wound repair-induced expression of a stromelysins is associated with the acquisition of a mesenchymal phenotype in human respiratory epithelial cells. Lab Invest 1996;74:658-669.

39. Gilles C, Polette M, Zahm JM, et al. Vimentin contributes to human mammary epithelial cell migration. J Cell Sci 1999;112: 4615-4625.

40. Gilles $C$, Polette $M$, Mestdagt $M$, et al. Transactivation of vimentin by beta-catenin in human breast cancer cells. Cancer Res 2003; 63:2658-2664. 